\title{
Retrograde recanalization of chronic total occlusion. A novel maneuver of the old technique
}

\author{
Mariusz Dębski ${ }^{1}$, Artur Dębski ${ }^{2}$, Paweł Tyczyński², Adam Witkowski², Marcin Demkow ${ }^{1}$ \\ ${ }^{1}$ Department of Coronary and Structural Heart Diseases, Institute of Cardiology, Warsaw, Poland \\ ${ }^{2}$ Department of Interventional Cardiology and Angiology, Institute of Cardiology, Warsaw, Poland
}

Adv Interv Cardiol 2017; 13, 1 (47): 82-83

DOI: https://doi.org/10.5114/aic.2017.66193

Percutaneous coronary intervention $(\mathrm{PCl})$ of chronic total occlusion (CTO) remains one of the most challenging procedures in interventional cardiology. In case of anterograde failure to cross the CTO lesion, the retrograde approach may improve the success rate of such procedures [1]. Factors predicting procedural success have been described before [2]. Thus, various devices and tips and tricks have been developed to further improve this strategy [3]. We present a novel wiring maneuver that enabled the operator to successfully complete the procedure.

A 52-year-old patient with a history of previous $\mathrm{PCl}$ of the right and the left circumflex coronary arteries, nicotine use, hypertension and dyslipidemia was admitted for a percutaneous attempt to open the CTO of the proximal left anterior descending coronary artery (LAD) (Figure $1 \mathrm{~A}$ ). Single-photon emission computed tomography revealed $20 \%$ inducible ischemia of the anterior wall. The left femoral artery was punctured and the left coronary artery was cannulated with an EBU 7 Fr guide catheter (GC). The anterograde approach with standard workhorse guidewires was unsuccessful. Virtually no LAD stump was visualized; therefore no CTO-dedicated wires were used and a retrograde approach was attempted. Sion (Asahi Intecc, Japan) guidewire with a Finecross (Terumo, Japan) $130 \mathrm{~cm}$ microcatheter were advanced retrogradely up to the LAD occlusion via the epicardial LCx collateral. After unsuccessful attempts to cross the CTO with Fielder FC, XT (Asahi Intecc), and Miracle Bross 12 (Asahi Intecc) guidewires, it was finally opened with a $180 \mathrm{~cm}$ Confianza PRO 12 (Asahi Intecc) (Figure $1 \mathrm{~B}$ ). The wire was then advanced retrogradely into the GC (up to the level of the aortic arch). Finecross microcatheter turned out to be too short to cross the occlusion and reach the GC retrogradely. Thus, an over-the wire (OTW) balloon was placed into the GC and inserted within it over the Confianza guidewire tip and further advanced anterogradely up to the CTO lesion (Figures 1 D, E). The Finecross microcatheter was then withdrawn and the retrograde wire was exchanged for an antegrade Fielder FC. The next steps of CTO PCI were straightforward. After predilatation two drug-eluting stents were implanted. A good angiographic result was achieved (Figure $1 \mathrm{~F}$ ). The postprocedural course was uneventful.

Subsequently we performed a bench study of cannulation of an OTW balloon using a Confianza wire within the $7 \mathrm{~F} \mathrm{GC}$. This technique proved to be achievable, although not very easy. Moreover, it was impossible to cannulate the OTW balloon using a soft tip wire. The crucial part of the maneuver itself is positioning the OTW balloon on the intraluminal exterior curvature of the GC and then using the GC wall as support while introducing the wire tip into the balloon.

Such an approach enables the operator to evade wire externalization. It may not be considered a method of choice when the strategy of the procedure is developed, but it may be helpful as a bailout in a variety of cases. In contrast to the "Rendezvous" method, where two microcatheters' ends face each other in the guide catheter, we did not use any microcatheter. The novelty of our approach is to use one guidewire and the OTW balloon opposite it, without any additional support. In conclusion, the technique described above may prove useful with retrograde CTO treatment.

\section{Conflict of interest}

The authors declare no conflict of interest. 

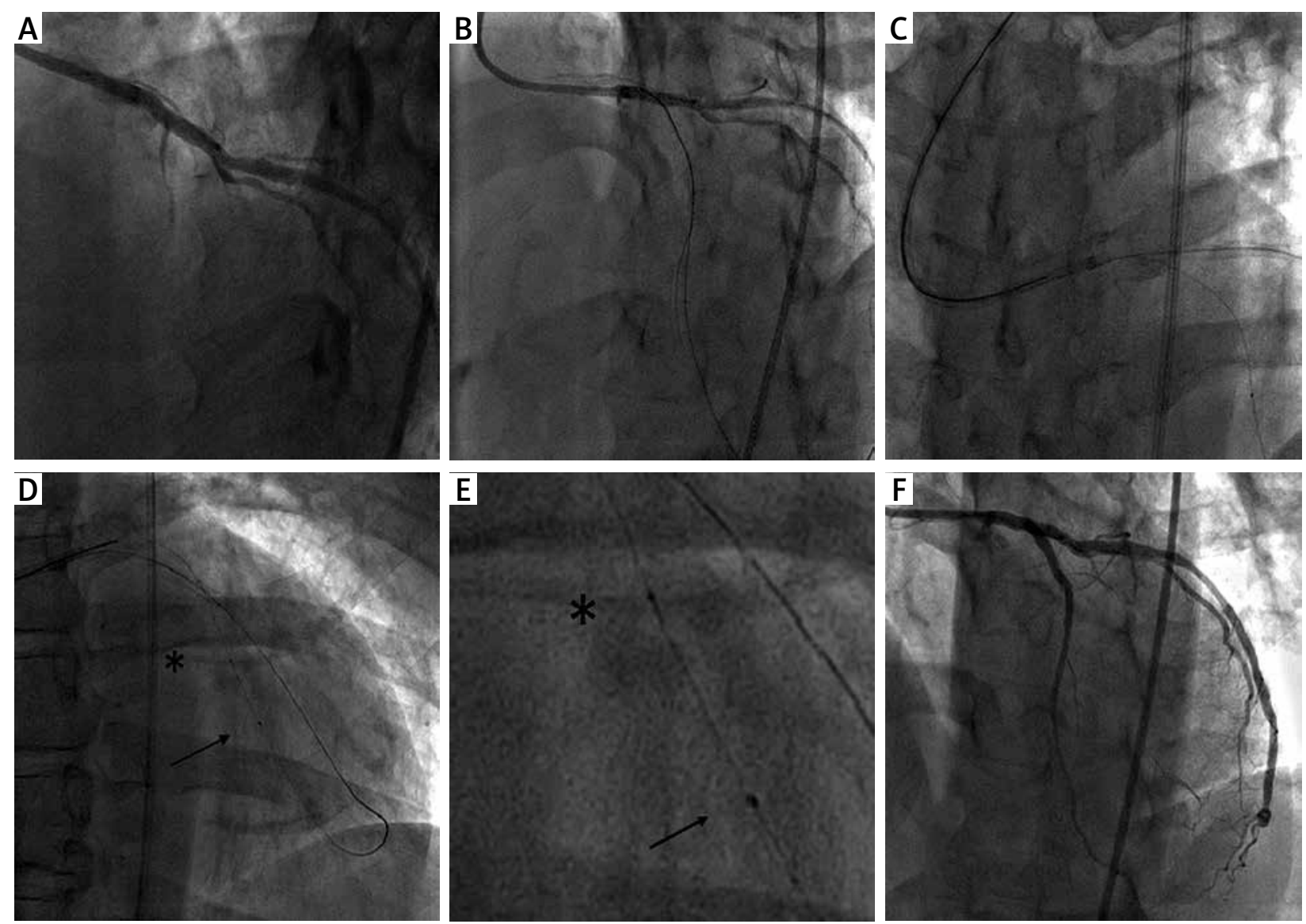

Figure 1. A - Baseline angiogram. Left anterior descending (LAD) artery closed in proximal segment. B - Chronic total occlusion (CTO) crossed retrogradely. C - Retrograde guidewire inside the guide catheter. D - Guidewire with microcatheter retrograde (arrow) and over the wire (OTW) balloon antegrade (asterisk). E - Magnification of image "D". F - Final result

\section{References}

1. Sheiban I, Moretti C, Omede P, et al. The retrograde coronary approach for chronic total occlusions: mid-term results and technical tips and tricks. J Interv Cardiol 2007; 20: 466-73.

2. Baykan AO, Gür M, Acele A, et al. Predictors of successful percutaneous coronary intervention in chronic total coronary occlusions. Adv Interv Cardiol 2016; 12: 17-24.

3. Saito S. Different strategies of retrograde approach in coronary angioplasty for chronic total occlusion. Catheter Cardiovasc Interv 2008; 71: 8-19. 